M. K A L E C K I (Warszawa)

\title{
MODEL MECHANISTYCZNY ZJAWISKA LOSOWEGO
}

1. Zasady "gry zegarkowej“6. Wyobraźmy sobie $m$ zegarków, których tarcze mają obwód równy jedności i są podzielone na dwa wycinki: jeden czarny, a drugi biały. Każdy z zegarków ma tylko jedną wskazówkę, której koniec wiruje z szybkością stałą. $\theta_{1}, \theta_{2}, \ldots, \theta_{m}$. Co jednostkę czasu sprawdzamy na każdym z $m$ zegarków, czy wskazówka jest w polu czarnym czy też $w$ białym. Jeśli liczba czarnych pól jest parzysta, notujemy $A$, jeśli jest nieparzysta - $B$. Jest to jedna partia. Gra polega na zgadywaniu, czy wydarzy się $A$ czy $B$. Zakładamy, że szybkości $\theta_{1}, \theta_{2}$, $\ldots, \theta_{m}$ są niewymierne i że są arytmetycznie niezależne (tzn. że żadne $\theta$ nie jest funkcja liniową o współczynnikach wymiernych pozostałych $\theta$ ).

Przyjmijmy za zero położenie końców wskazówek w czasie zero. Wtedy położenia te w kolejnych $N$ partiach będą:

$$
\begin{aligned}
& \theta_{1}-\left[\theta_{1}\right], \quad 2 \theta_{1}-\left[2 \theta_{1}\right], \quad \ldots, \quad N \theta_{1}-\left[N \theta_{1}\right], \\
& \theta_{2}-\left[\theta_{2}\right], \quad 2 \theta_{2}-\left[2 \theta_{2}\right], \quad \ldots, \quad N \theta_{2}-\left[N \theta_{2}\right], \\
& \theta_{m}-\left[\theta_{m}\right], \quad 2 \theta_{m}-\left[2 \theta_{m}\right], \quad \ldots, \quad N \theta_{m}-\left[N \theta_{m}\right] \text {, }
\end{aligned}
$$

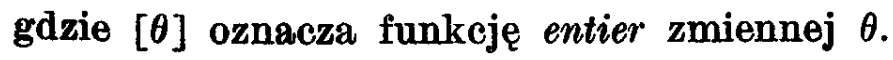

Należy zaznaczyć, że jednoczéśnie z grą opartą na zespole $m$ zegarków można prowadzić gry na tych samych zasadach w odniesieniu do każdego podzespohu pierwszych $k$ zegarków.

2. Częstości występowania wyników $A$ i $B$. Wprowadźmy następujæce oznaczenia: częstosć występowania białego pola na $k$-tym zegarku oznaczmy przez $b_{N k}$, a czarnego pola przez $c_{N k}$; dalej oznaczmy dhugość łuku wycinka białego na $k$-tym zegarku przez $p_{k}$, a więc długośc łuku wycinka czarnego będzie $1-p_{k}$; wreszcie częstości występowania liter $A$ i $B$ w grze opartej na podzespole pierwszych $k$ zegarków oznaczmy przez $(A)_{N k}$. Wobec tego częstości występowania $A$ i $B$ w grze opartej na wszystkich $m$ zegarkach oznaczać będziemy odpowiednio przez $(A)_{N m}$ i $(B)_{N m}$. 
TWIERDzENIE 1. Jeśli $\left|1-2 p_{k}\right| \leqslant \alpha<1$, gdzie a jest liczba stalq oraz $k=1,2, \ldots, m$, to $\lim _{m \rightarrow \infty} \lim _{N \rightarrow \infty}(A)_{N m}=\lim _{m \rightarrow \infty} \lim _{N \rightarrow \infty}(B)_{N m}=\frac{1}{2}$.

Dowód. Z twierdzeń w aneksie (str. 123) wynikaja następujące właściwości wskazań zegarków. Jeśli, jak założono, szykości $\theta_{1}, \theta_{2}, \ldots, \theta_{m}$ są niewymierne $\mathrm{i}$ żadna $z$ nich nie jest funkeja liniową o współczynnikach wymiernych pozostałych $\theta$, to mamy:

(1) Według twierdzenia I aneksu:

$$
\lim _{N \rightarrow \infty} b_{N k}=p_{k}, \quad \lim _{N \rightarrow \infty} c_{N k}=1-p_{k}
$$

(2) Według twierdzenia II aneksu częstość występowania w jednej partii kombinacji pola bialego na zegarku $k$ i pola bialego na zegarku $l$ zmierza do $p_{k} p_{l}$, gdy $N \rightarrow \infty$. Granice częstości dla kombinacji białe-czarne oraz czarne-białe czy téz wreszcie czarne-czarne będą odpowiednio $p_{k}\left(1-p_{l}\right),\left(1-p_{k}\right) p_{l}$ oraz $\left(1-p_{k}\right),\left(1-p_{l}\right)$. Analogiczne twierdzenie będzie słuszne dla kombinacji obejmującej dowolny podzespół zegarków czy téz wszystkie $m$ zegarków. Używając terminologii rachunku prawdopodobieństwa możemy powiedzieć, że wskazanie $k$-tego zegarka jest asymptotycznie niezależne od wskazań innych.

Weźmy teraz pod uwage grę opartą na podzespole pierwszych $k$ zegarków. Z właściwości (1) i (2) wynika, że $(A)_{N k}$ zmierza do określonej granicy $(A)_{k}$, a $(B)_{N k}$ do $(B)_{k}$, gdy $N \rightarrow \infty$.

Dolączmy teraz do podzespołu $(k+1)$-szy zegarek. W grze opartej na tym nowym podzespole $A$ wystąpi w danej partii: albo jeśli w grze opartej na podzespole pierwszych $k$ zegarków wystąpiło w tej partii $A$ i na $(k+1)$-szym zegarku wskazówka zatrzymała sie w białym polu, albo jeśli $w$ grze opartej na podzespole pierwszych $k$ zegarków wystapiło w tej partii $B$ i na $(k+1)$-szym zegarku wskazówka zatrzymała się w czarnym polu.

Biorąc pod uwage ,niezależność" partyj na poszczególnych zegarkach, znajdujemy dla granicznej częstości $(A)_{k+1}$ :

Analogicznie

$$
(A)_{k+1}=(A)_{k}\left(1-p_{k+1}\right)+(B)_{k} p_{k+1} .
$$

Wobec tego

$$
(B)_{k+1}=(B)_{k}\left(1-p_{k+1}\right)+(A)_{k} p_{k+1} \text {. }
$$

$$
(A)_{k+1}-(B)_{k+1}=\left((A)_{k}-(B)_{k}\right)\left(1-2 p_{k+1}\right) .
$$

Za pomoca indukcji zupełnej otrzymujemy

$$
(A)_{m}-(B)_{m}=\left(1-2 p_{1}\right)\left(1-2 p_{2}\right) \ldots\left(1-2 p_{m}\right) .
$$


Z założenia $\left|1-2 p_{k}\right| \leqslant \alpha<1$ wynika, że

Mamy więc

$$
\lim _{m \rightarrow \infty}\left((A)_{m}-(B)_{m}\right)=0 \text {. }
$$

albo

$$
\lim _{m \rightarrow \infty}(A)_{m}=\lim _{m \rightarrow \infty}(B)_{m}=\frac{1}{2}
$$

$$
\lim _{m \rightarrow \infty} \lim _{N \rightarrow \infty}(A)_{N m}=\lim _{m \rightarrow \infty} \lim _{N \rightarrow \infty}(B)_{N m}=\frac{1}{2},
$$

i to przy dowolnych wartosciach $p_{1}, p_{2}, \ldots, p_{m}$, jesli tylko spełniony jest warunek $\left|1-2 p_{k}\right| \leqslant a<1$.

3. "Niezależnośé" dwóch kolejnych wyników gry. Oznaczmy cęzstość zmiany z czarnego na białe, lub vice versa, $\mathrm{w}$ dwóch kolejnych partiach (tzn. wystepowania kombinacji białe-czarne lub czarne-białe) na $k$-tym zegarku przez $\delta_{N k}$. Częstosć pozostawania białego lub czarnego bez zmiany w dwóch kolejnych partiach (tzn. występowania kombinacji białe- białe lub czarne-czarne) będzie więc równa $1-\delta_{N k}$. Dalej oznaczmy częstości występowania kombinacji $A A, B B, A B$ i $B C$ w dwóch kolejnych partiach dla gry opartej na podzespole pierwszych $k$ zegarków odpowiednio przez $(A A)_{N k},(B B)_{N k},(A B)_{N k}$ i $(B A)_{N k}$. Wobec tego częstości występowania $A A, B B, A B$ i $B A$ w dwoch kolejnych partiach dla gry opartej na wszystkich $m$ zegarkach oznaczać będziemy odpowiednio przez $(A A)_{N m}$, $(B B)_{N m},(A B)_{N m} \mathrm{i}(B A)_{N m}$. Oznaczmy wreszcie przez $Z_{N k}$ częstość zmiany z $A$ na $B$ lub vice versa w dwóch kolejnych partiach dla gry opartej na pierwszych $k$ zegarkach; mamy więc

$$
Z_{N k}=(A B)_{N k}+(B A)_{N k}, \quad 1-Z_{N k}=(A A)_{N k}+(B B)_{N k} .
$$

Dla gry opartej na wszystkich $m$ zegarkach mamy oczywiscie

$$
Z_{N m}=(A B)_{N m}+(B A)_{N m}, \quad 1-Z_{N m}=(A A)_{N m}+(B B)_{N m} .
$$

Wreszcie oznaczmy $\theta_{k}-\left[\theta_{k}\right]$ przez $\theta_{k}^{\prime}$.

TwIERdzenIe 2. Jeśli $\left|1-2 p_{k}\right| \leqslant \alpha<1$ oraz $\theta_{k} \geqslant \beta>0,1-\theta_{k} \geqslant$ $\geqslant \beta>0 i\left|\frac{1}{2}-\theta_{k}^{\prime}\right| \geqslant \beta>0$, gdzie $\alpha i \beta$ sq liczbami statymi, to $\lim _{m \rightarrow \infty} \lim _{N \rightarrow \infty}(A A)_{N m}=\lim _{m \rightarrow \infty} \lim _{N \rightarrow \infty}(B B)_{N m}=\lim _{m \rightarrow \infty} \lim _{N \rightarrow \infty}(A B)_{N m}=\lim _{m \rightarrow \infty} \lim _{N \rightarrow \infty}(B A)_{N m}$.

Dowód. Z twierdzeń aneksu wynikają następujące właściwości wskazań zegarków:

(3) Wedhug twierdzenia III aneksu $\delta_{N k}$ zmierza do pewnej granicy $\delta_{k}$, gdy $N \rightarrow \infty$, czyli

$$
\lim _{N \rightarrow \infty} \delta_{N k}=\delta_{k} \quad \text { oraz } \quad \lim _{N \rightarrow \infty}\left(1-\delta_{N k}\right)=1-\delta_{k} .
$$


Przy tym wedhug twierdzenia $V$ aneksu, jeżeli $\left|1-2 p_{k}\right| \leqslant \alpha<1$ oraz $\theta_{k}^{\prime} \geqslant \beta>0,1-\theta_{k}^{\prime}>\beta>0$ i $\left|\frac{1}{2}-\theta_{k}^{\prime}\right| \geqslant \beta>0$, gdzie $\alpha$ i $\beta$ są liczbami stałymi, to $\left|1-2 \delta_{k}\right| \leqslant \lambda<1$, gdzie $\lambda$ jest znown liczbą stałą.

(4) Według twierdzenia IV aneksu częstość zdarzenia, ,zmiana" (lub zdarzenia ,bez zmiany") w dwu kolejnych wskazaniach $k$-tego zegarka jest „niezależna" od częstości ,zmiany" (lub „,bez zmiany”) na innych zegarkach. Np. graniczna częstość kombinacji „zmiany" na $k$-tym zegarku i ,bez zmiany" na $l$-tym zegarku wynosi $\delta_{k}\left(1-\delta_{l}\right)$.

Weźmy teraz pod uwage gre oparta na podzespole pierwszych $k$ zegarków. Z właściwości (3) i (4) wynika, że jeśli $N \rightarrow \infty$, to $Z_{N k}$ i $1-Z_{N k}$ zmierzają do określonych granic, które oznaczymy odpowiednio przez $Z_{k}$ i $1-Z_{k}$.

Dołączmy do podzespołu $(k+1)$-sży zegarek. W grze opartej na tym nowym podzespole ,zmiana" nastąpi $w d w u$ kolejnych partiach $w d w u$ przypadkach :

$1^{\circ}$ jeśli w grze opartej na zespole $k$ pierwszych zegarków wystąpiła ,zmiana", a na $(k+1)$-szym zegarku wystąpiło ,bez zmiany"; wtedy bowiem w pierwszej $\mathrm{z}$ dwu kolejnych partii mamy $\mathrm{w}$ zespole pierwszych $k$ zegarków nieparzystą ilość ,czarnych", a w drugim parzystą ilość ,czarnych", lub vice versa, a na $(k+1)$-szym zegarku mamy dwa ,czarne" lub dwa ,białe";

$2^{\circ}$ jeśli w grze opartej na podzespole pierwszych $k$ zegarków wystąpiło „bez zmiany", a na $(k+1)$-szym zegarku ,zmiana"; wtedy bowiem w obu kolejnych partiach $\overline{\mathrm{w}}$ zespole pierwszych $k$ zegarków mamy parzystą lub nieparzysta ilość „czarnych", a na $(k+1)$-szym zegarku mamy ,czarne-białe" lub ,białe-czarne".

Biorąc pod uwage „niezależność" zdarzeń „zmiana” i ,bez zmiany” we wskazaniach poszczególnych zegarków znajdujemy stąd dla granicznej częstości ,zmiany"

$$
Z_{k+1}=Z_{k}\left(1-\delta_{k+1}\right)+\left(1-Z_{k}\right) \delta_{k+1} .
$$

Analogieznie otrzymujemy

$$
1-Z_{k+1}=\left(1-Z_{k}\right)\left(1-\delta_{k+1}\right)+Z_{k} \delta_{k+1} .
$$

Stąd wynika, że $2 Z_{k+1}-1=\left(1-2 \delta_{k+1}\right)\left(2 Z_{k}-1\right)$ i, drogą indukcji zupelnej,

$$
1-2 Z_{m}=\left(1-2 \delta_{1}\right)\left(1-2 \delta_{2}\right) \ldots\left(1-2 \delta_{m}\right) \text {. }
$$

Ponieważ $\left|1-2 \delta_{k}\right| \leqslant \lambda<1$, gdzie $\lambda$ jest liczbą stałฉ, otrzymujemy

$$
\lim _{m \rightarrow \infty}\left(1-2 Z_{m}\right)=0, \quad \text { czyli } \quad \lim _{m \rightarrow \infty} Z_{m}=\frac{1}{2} \text {. }
$$


Możemy teraz wykazać, ̇̇e częstości występowania kombinacyj $A A, B B, A B$ i $B A$ w dwóch kolejnych partiach gry, opartej na wszystkich $m$ zegarkach, zmierzają do granicy $\frac{1}{4}$, gdy $N \rightarrow \infty$ i $m \rightarrow \infty$. Mamy przede wszystkim $(A B)_{N m}+(B A)_{N m}=Z_{N m}$. Stąd wynika, że

$$
\lim _{N \rightarrow \infty}\left((A B)_{N m}+(B A)_{N m}\right)=Z_{m} .
$$

Dalej mamy

$\lim _{N \rightarrow \infty}\left((A B)_{N m}+(A A)_{N m}\right)=(A)_{m} \quad$ oraz $\quad \lim _{N \rightarrow \infty}\left((B A)_{N m}+(A A)_{N m}\right)=(A)_{m}$, czyli

$$
\lim _{N \rightarrow \infty}\left((A B)_{N m}-(B A)_{N m}\right)=0 .
$$

Otrzymujemy więc

$$
\lim _{N \rightarrow \infty}(A B)_{N m}=\lim _{N \rightarrow \infty}(B A)_{N^{\prime} m}=\frac{1}{2} Z_{m} .
$$

Ponieważ wykazaliśmy wyżej, że $\lim _{m \rightarrow \infty} Z_{m}=\frac{1}{2}$, otrzymujemy

$$
\lim _{m \rightarrow \infty} \lim _{N \rightarrow \infty}(A B)_{N m}=\lim _{m \rightarrow \infty} \lim _{N \rightarrow \infty}(B A)_{N m}=\frac{1}{4} .
$$

Biorąc pod uwage, że $(A A)_{N m}+(B B)_{N m}+(A B)_{N m}+(B A)_{N m}=1$, można z łatwościa wykazać, że również

$$
\lim _{m \rightarrow \infty} \lim _{N \rightarrow \infty}(A A)_{N m}=\lim _{m \rightarrow \infty} \lim _{N \rightarrow \infty}(B B)_{N m}=\frac{1}{4},
$$

czyli dwie kolejne partie w grze opartej na $m$ zegarkach sa od siebie asymptotycznie ,niezależne" $z$ dowolnym przybliżeniem, jeśli $m$ jest dostatecznie wielkie.

4. Uogólnienia. Najpierw zajmiemy się ,niezależnością” j-tego i $(j+a)$-tego wyniku gry.

Niech a będzie określoną liczbą całkowitą i dodatnią. Wprowadźmy następujace oznaczenia. Częstość zmiany z czarnego na biale lub vice versa we wskazaniach $j$ i $j+a$ na $k$-tym zegarku oznaczmy przez $\delta_{N k}^{(a)}$. Czesstość pozostawania białego lub czarnego we wskazaniach $j \mathrm{i} j+a$ będzie więc równa $1-\delta_{N k}^{(a)}$. Dalej oznaczmy przez $A_{j}$ i $B_{j}$ zjawiska polegające na występoẉaniu litery $A$ lub $B$ na $j$-tym miejscu w ciaggu liter $A$ i $B$. Częstości wystepowania kombinacyj $A A, B B, A B$ i $B A$ na miejscach $j$ i $j+a$ w grze opartej na $k$ pierwszych zegarkach oznaczymy przez $\left(A_{j} A_{j+a}\right)_{N k},\left(B_{j} B_{j+a}\right)_{N k}$, $\left(A_{j} B_{j+a}\right)_{N k}$ oraz $\left(B_{j} A_{j+a}\right)_{N k}$; w grze zaś opartej na wszystkich $m$ zegarkach - przez $\left(A_{j} A_{j+a}\right)_{N m},\left(B_{j} B_{j+a}\right)_{N m},\left(A_{j} B_{j+a}\right)_{N m}$ oraz $\left(B_{j} A_{j+a}\right)_{N m}$. Wreszcie oznaczmy $a \theta_{k}-\left[a \theta_{k}\right]$ przez $\theta_{k}^{(a)}$. 
TWIERDZENIE 3. Jeśli $\left|1-2 p_{k}\right| \leqslant \alpha<1$ oraz $\theta_{k}^{(a)} \geqslant \beta^{(a)}>0,1-\theta_{k}^{(a)} \geqslant$ $\geqslant \beta^{(a)}>0$ i $\left|\frac{1}{2}-\theta_{k}^{(a)}\right| \geqslant \beta^{(a)}>0$, gdzie a $i \beta^{(a)}$ sa liczbami stalymi, to

$$
\begin{aligned}
\lim _{m \rightarrow \infty} \lim _{N \rightarrow \infty}\left(A_{j} A_{j+a}\right)_{N m} & =\lim _{m \rightarrow \infty} \lim _{N \rightarrow \infty}\left(B_{j} B_{j+a}\right)_{N m}= \\
& =\lim _{m \rightarrow \infty} \lim _{N \rightarrow \infty}\left(A_{j} B_{j+a}\right)_{N m}=\lim _{m \rightarrow \infty} \lim _{N \rightarrow \infty}\left(B_{j} A_{j+a}\right)_{N m}=\frac{1}{4} .
\end{aligned}
$$

Dowód. Z twierdzenia VI aneksu wynikają następujące uogólnienia właściwości (3) i (4) wskazań zegarków:

$\delta_{N k}^{(a)}$ zmierza do pewnej granicy $\delta_{k}^{(a)}$, gdy $N \rightarrow \infty$, czyli

$$
\lim _{N \rightarrow \infty} \delta_{N k}^{(a)}=\delta_{k}^{(a)} \text {. }
$$

Przy tym, jeśli $\left|1-2 p_{k}\right| \leqslant \alpha<1$ oraz $\theta_{k}^{(a)} \geqslant \beta^{(a)}>0,1-\theta_{k}^{(a)} \geqslant \beta^{(a)}>0$, i $\left|\frac{1}{2}-\theta_{k}^{(a)}\right| \geqslant \beta^{(a)}>0$, gdzie $\alpha$ i $\beta^{(a)}$ sa liczbami stalymi, to $\left|1-2 \delta_{k}^{(a)}\right| \leqslant$ $\leqslant \lambda^{(a)}<1$, gdzie $\lambda^{(a)}$ jest znowu liczbą stałą.

(4') Częstość zdarzenia ,zmiana" (lub ,bez zmiany") we wskazaniu $j$-tym i $(j+a)$-tym na $k$-tym zegarku jest niezależna od częstości „zmiany" na innych zegarkach. Np. graniczna częstość kombinacji "zmiany" na $k$-tym zegarku i ,bez zmiany" na $l$-tym zegarku we wskazaniach $j$-tym i $(j+a)$-tym wynosi $\delta_{k}^{(a)}\left(1-\delta_{2}^{(a)}\right)$.

- Na podstawie tych właściwości można przeprowadzić dowód w ten sam sposób, jak dla poprzedniego twierdzenia.

Dalsze uogólnienie dotyczy jednoczesnej niezależności wyników $j+1, j+2, \ldots, j+a$ od wyniku $j$-tego.

Oznaczmy najmniejszą wielokrotność liczb naturalnych niewiększych od $a$ przez $w$ oraz $w \theta_{k}-\left[w \theta_{k}\right]$ przez $\theta_{k}^{(w)}$.

TwIERDZENIE 4. Jeśli $\left|1-2 p_{k}\right| \leqslant \alpha<1$ oraz $\theta_{k}^{(w)} \geqslant \beta^{(w)}>0,1-\theta_{k}^{(w)} \geqslant$ $\geqslant \beta^{(w)}>0 i\left|\frac{1}{2}-\theta_{k}^{(w)}\right| \geqslant \beta^{(w)}>0$, gdzie a $i \beta^{(w)}$ sa liczbami stalymi, to

$$
\begin{aligned}
\lim _{m \rightarrow \infty} \lim _{N \rightarrow \infty}\left(A_{j} A_{j+i}\right)_{N m} & =\lim _{m \rightarrow \infty} \lim _{N \rightarrow \infty}\left(B_{j} B_{j+i}\right)_{N m}=\lim _{m \rightarrow \infty} \lim _{N \rightarrow \infty}\left(A_{j} B_{j+i}\right)_{N m}= \\
& =\lim _{m \rightarrow \infty} \lim _{N \rightarrow \infty}\left(B_{j} A_{j+i}\right)_{N m}=\frac{1}{4} \quad(i=1,2, \ldots, a) .
\end{aligned}
$$

Dowód. Z twierdzenia 3 wynika, że twierdzenie 4 jest słuszne, jeśl $1^{i}$ spełnione sa jednocześnie warunki (a), (b) i (c):

$$
\begin{aligned}
j \theta_{k}-\left[j \theta_{k}\right] & \geqslant \beta^{(i)}>0, \\
1-j \theta_{k}+\left[j \theta_{k}\right] & \geqslant \beta^{(i)}>0, \\
\left|\frac{1}{2}-j \theta_{k}+\left[j \theta_{k}\right]\right| & \geqslant \beta^{(i)}>0,
\end{aligned}
$$

gdzie $\beta^{(i)}(i=1,2, \ldots, a)$ są to liczby stałe. Otóż dowiedziemy, $\dot{z} \Theta$ warunki te sa spełnione dla $\beta^{(i)}=i \beta^{(w)} / w$. Mamy przede wszystkim 
$\left(a^{\prime}\right)$

$\left(\mathbf{c}^{\prime}\right)$

$$
\begin{aligned}
w \theta_{k}-\left[w \theta_{k}\right] & \geqslant \beta^{(w)}>0, \\
1-w \theta_{k}+\left[w \theta_{k}\right] & \geqslant \beta^{(w)}>0, \\
\left|\frac{1}{2}-w \theta_{k}+\left[w \theta_{k}\right]\right| & \geqslant \beta^{(w)}>0 .
\end{aligned}
$$

Zacznijmy od warunku (a). Możemy oczywiście napisać

$$
i \theta_{k}-\left[i \theta_{k}\right]>0
$$

(ponieważ $\theta_{k}$ jest liczbą niewymierną). Pomnóżmy obie strony tej nierówności przez wyrażenie $w / i$, które jest liczba całkowita, gdyż $w$ jest wielokrotnością $i$ :

$$
w \theta_{k}-\frac{w}{i}\left[i \theta_{k}\right]>0
$$

Ponieważ $\left[w \theta_{k}\right]$ jest największą liczbą calkowita zawarta w $w \theta_{k}$, mamy

$$
w \theta_{k}-\frac{w}{i}\left[i \theta_{k}\right] \geqslant w \theta_{k}-\left[w \theta_{k}\right]
$$

Stąd i z (a') wynika, że

$$
w \theta_{k}-\frac{w}{i}\left[i \theta_{k}\right] \geqslant \beta^{(w)},
$$

a dzieląc przez $w / i$ otrzymujemy

$$
i \theta_{k}-\left[i \theta_{k}\right] \geqslant \frac{i}{w} \beta^{(w)}>0
$$

co dowodzi spełnienia warunku (a).

Rozpatrzmy teraz warunek (b). Mamy $1-i \theta_{k}+\left[i \theta_{k}\right]>0$. Pomnóżmy obie strony tej nierówności przez $w / i$ :

$$
\frac{w}{i}-w \theta_{k}+\frac{w}{i}\left[i \theta_{k}\right]>0 \text {. }
$$

Ponieważ $\left[w \theta_{k}\right]+1$ jest najmniejszą liczbą całkowita większq od $w \theta_{k}$, mamy

$$
\frac{w}{i}-w \theta_{k}+\frac{w}{i}\left[i \theta_{k}\right] \geqslant 1-w \theta_{k}+\left[w \theta_{k}\right]
$$

Stąd i z (b') wynika, że

$$
\frac{w}{i}-w \theta_{k}+\frac{w}{i}\left[i \theta_{k}\right] \geqslant \beta^{(w)}>0 ;
$$


dzieląc przez $w / i$ otrzymujemy

$$
1-i \theta_{k}+\left[i \theta_{k}\right] \geqslant \frac{i}{w} \beta^{(w)}>0
$$

co dowodzi spełnienia warunku (b).

Wreszcie dla warunku (c) dowód przedstawia się jak następuje.

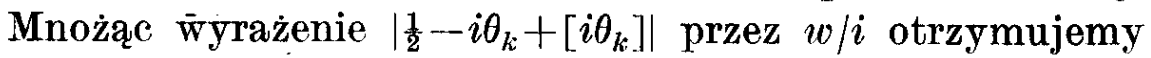

$$
\frac{w}{2 i}-w \theta_{k}+\frac{w}{i}\left[i \theta_{k}\right]
$$

Ponieważ albo $\frac{1}{2}+\left[w \theta_{k}\right]$, albo $1+\left[w \theta_{k}\right]$ jest najbliższa do $w \theta_{k}$ wielokrotnościa liczby $\frac{1}{2}$, więc mamy albo

$$
\left|\frac{w}{2 i}-w \theta_{k}+\frac{w}{i}\left[i \theta_{k}\right] \geqslant\right| \frac{1}{2}-w \theta_{k}+\left[w \theta_{k}\right] \mid
$$

albo

$$
\frac{w}{2 i}-w 0_{k}+\frac{w}{i}\left[i \theta_{k}\right] \geqslant 1-w \theta_{k}+\left[w \theta_{k}\right]
$$

Ale według założeń $\left(b^{\prime}\right)$ i $\left(c^{\prime}\right)$ wyrażenia po prawej stronie obu tych nierówności są niemniejsze od $\beta^{(w)}$, które jest dodatnie. Wobec tego

$$
\left|\frac{w}{2 i}-w \theta_{k}+\frac{w}{i}\left[i \theta_{k}\right]\right| \geqslant \beta^{(w)}>0
$$

i po podzieleniu przez $w / i$ otrzymujemy

$$
\left|\frac{1}{2}-i \theta_{k}+\left[i \theta_{k}\right]\right| \geqslant \frac{i}{w} \beta^{(w)}>0
$$

co dowodzi spełnienia warunku (c).

Twierdzenie 4 sformułować można również jak następuje:

Wynik gry zegarkowej jest ,niezależny" od wyników poprzednich a partyj $z$ dowolnym przybliżeniem, gdy $N i m$ sq dostatecznie wielkie, jeśli $\theta_{k}^{(w)}, 1-\theta_{k}^{(w)}$ oraz $\left|\frac{1}{2}-\theta_{k}^{(w)}\right|$ sq wieksze od pewnej stalej dodatniej, gdzie $\theta_{k}^{(v)}=$ $=w \theta_{k}-\left[w \theta_{k}\right], a w$ jest najmniejsza wielokrotnosicia liczb naturalnych nieviekteszych od $a$.

Należy zaznaczyć, że warunki ograniczające dla zespołu $\theta_{k}$ zależą od a. To samo dotyczy wielkości $m$, niezbędnej do danego przybliżenia do "niezależności" wyniku gry od a poprzednich wyników.

Jak widać z powyższego, możemy dowolnie upodobnić wyniki gry zegarkowej do ciagu losowego. Zakładamy przede wszystkim dowolnie 
duże, ale określone $a$. Dobierzmy zespół $m$ liczb niewymiernych $\varphi_{1}, \varphi_{2}, \ldots$, $\varphi_{m}$ arytmetycznie niezależnych, spełniających warunki

$$
\varphi_{k}-\left[\varphi_{k}\right]>\beta^{(w)}, \quad 1-\varphi_{k}+\left[\varphi_{k}\right]>\beta^{(w)} \quad \text { oraz } \quad\left|\frac{1}{2}-\varphi_{k}+\left[\varphi_{k}\right]\right|>\beta^{(w)},
$$

gdzie $\beta^{(w)}$ jest stalą liczbą dodatnią. Przyjmijmy za szybkości końców wskaź́wek $m$ zegarków liczby $\varphi_{1} / w, \varphi_{2} / w, \ldots, \varphi_{m} / w$, gdzie $w$ jest najmniejszą wielokrotnościa liczb naturalnych niewiększych od a. Przy dostatecznie dużym $m$ wynik gry zegarkowej będzie „niezależny” od poprzednich $a$ 'wyników $\mathrm{z}$ dowolnych przybliżeniem.

Warto jeszcze zwrócić uwage na szczególny przypadek spelnienia przez zespół $\theta_{k}$ warunków

$$
\begin{aligned}
w \theta_{k}-\left[w \theta_{k}\right] & >\beta^{(w)}>0, \\
1-w \theta_{k}+\left[w \theta_{k}\right] & >\beta^{(w)}>0, \\
\left|\frac{1}{2}-w \theta_{k}+\left[w \theta_{k}\right]\right| & >\beta^{(w)}>0 .
\end{aligned}
$$

Można mianowicie wykazać, że warunki te sa spełnione, gdy $\theta_{1}$, $\theta_{2}, \ldots, \theta_{m}$ leżą w dostatecznie małym przedziale. Oznaczmy długość tego przedziału przez $\varepsilon^{(w)}$. Mamy wtedy $\left|\theta_{k}-\theta_{1}\right| \leqslant \varepsilon^{(w)}$. Niech $\varepsilon^{(w)}$ będzie dostatecznie małe, by spełniało warunki

$$
\begin{aligned}
& w \varepsilon^{(i v)}<w \theta_{1}-\left[w \theta_{1}\right], \\
& w \varepsilon^{(w)}<1-w \theta_{1}+\left[w \theta_{1}\right], \\
& w \varepsilon^{(w)}<\left|\frac{1}{2}-w \theta_{1}+\left[w \theta_{1}\right]\right| .
\end{aligned}
$$

Wynika stąd, że

$$
\left|w \theta_{k}-w \theta_{1}\right|<w \theta_{1}-\left[w \theta_{1}\right] \quad \text { oraz } \quad\left|w \theta_{k}-w \theta_{1}\right|<1-w \theta_{1}+\left[w \theta_{1}\right],
$$

a stąd z kolei

$$
\left[w \theta_{k}\right]=\left[w \theta_{1}\right]
$$

Otrzymujemy dalej

$$
\begin{aligned}
w \theta_{k}-\left[w \theta_{k}\right] & =w \theta_{1}-\left[w \theta_{1}\right]+w\left(\theta_{k}-\theta_{1}\right), \\
1-w \theta_{k}+\left[w \theta_{k}\right] & =1-w \theta_{1}+\left[w \theta_{1}\right]-w\left(\theta_{k}-\theta_{1}\right), \\
\left|\frac{1}{2}-w \theta_{k}+\left[w \theta_{k}\right]\right| & =\left|\frac{1}{2}-w \theta_{1}+\left[w \theta_{1}\right]-w\left(\theta_{k}-\theta_{1}\right)\right| .
\end{aligned}
$$

z warunku $\left|\theta_{k}-\theta_{1}\right| \leqslant \varepsilon^{(v)}$ wynika

$$
\begin{gathered}
w \theta_{k}-\left[w \theta_{k}\right] \geqslant w \theta_{1}-\left[w \theta_{1}\right]-w \varepsilon^{(\omega)}, \\
1-w \theta_{k}+\left[w \theta_{k}\right] \geqslant 1-w \theta_{2}+\left[w \theta_{1}\right]-w \varepsilon^{(w)}, \\
\left|\frac{1}{2}-w \theta_{k}+\left[w \theta_{k}\right]\right| \geqslant\left|\frac{1}{2}-w \theta_{1}+\left[w \theta_{1}\right]\right|-w \varepsilon^{(\omega)} .
\end{gathered}
$$


Z warunków (e), (f) i (g) dla $\varepsilon^{(w)}$ wynika z kolei, że wyrażenia po prawej stronie nierówności sa liczbami dodatnimi. Oznaczywszy najmniejszą z nich przez $\beta^{(w)}$ otrzymujemy właściwości $\left(\mathrm{a}^{\prime}\right),\left(\mathrm{b}^{\prime}\right)$ i $\left(\mathrm{c}^{\prime}\right)$.

Jak widać z powyższego, skupienie szybkości $\theta_{k} \mathrm{w}$ dostatecznie małym obszarze, zależnym od $a$, i dostatecznie wielka liczba $m$ zegarków stwarzaja warunki, w których wynik pewnej partii jest z dowolnym przybliżeniem niezależny od poprzednich a wyników. Latwo zauważyé, że warunki takie można zapewnić również przez skupienie się $\theta_{k}$ w skończonej ilości dostatecznie małych obszarów.

5. Model rouge et noir. Powyższe twierdzenia pozwola nam na skonstruowanie, bardzo zreszta prymitywnego, modelu wystepowania "czerwonego" i „,czarnego" w ruletce. Przyjmujemy, że ruch kulki zawsze w tym samym kierunku zaczyna się od punktu zerowego, leżącego na samym początku jakiejś działki, powiedzmy, czerwonej. Jeśli wobec tego oznaczymy przez $n$ liczbę działek zawartych w odległości przebytej przez kulkę, to wystąpienie $\mathrm{w}$ danej próbie czerwonego albo czarnego zależy od tego, czy $n$ jest parzyste, czy nieparzyste. Przyjmujemy również, że próby odbywają się w równych odstępach czasu.

-Wartość $n$ jest wynikiem zjawiska bardzo złożonego. Wyobraźmy sobie, że $n$ jest wyznaczane w kolejnych próbach przez nasz zespół z bardzo dużej liczby $m$ zegarków, mianowicie: $n$ równa się liczbie pól czarnych wskazywanych na zegarkach w chwili rozpoczęcia każdej próby. W ten sposób parzyste $n$, czyli wystąpienie rouge, odpowiada literze $A$ w grze zegarkowej, a nieparzyste $n$, czyli noir, odpowiada literze $B$.

Zakładamy, jak wyżej, że szybḱości końców wskazówek zegarków $\theta_{1}, \theta_{2}, \ldots, \theta_{m}$ są liczbami niewymiernymi i arytmetycznie niezależnymi. Zakładamy również, że spełniają one warunki niezbędne dla zapewnienia przybliżonej ,niezależności" wyniku gry zegarkowej od poprzednich $a$ wyników. Np. zakładamy, że szybkości $\theta_{1}, \theta_{2}, \ldots, \theta_{m}$ znajdują się w obrębie kilku dostatecznie małych obszarów.

Z dowiedzionych wyżej twierdzeń wynika, że w takim modelu:

$1^{0}$ częstość występowania czerwonego lub czarnego przy dostatecznie wielkiej ilości prób $N$ zbliża się dowolnie do pewnej liczby, która różni się bardzo mało od $\frac{1}{2}$, jeśli $m$, jak to założylismy wyżej, jest bardzo duże,

$2^{\circ}$ wynik próby jest ,niezależny", ze znacznym przybliżeniem; od wyników a poprzednich prób.

Oczywiście oba te zjawiska wystąpią równièz, jeśli $n$ jest wyznaczone przez jakikolwiek mechanizm posiadający podstawowe właśiwości zespohu $m$ zegarków. 


\section{$A N E K S$}

Pewne właściwości ciągów liczb niewymiernych $\theta, 2 \theta, 3 \theta ; \ldots$

DEFINICJA 1. Niech będzie dany ciąg liczb rzeczywistych

$$
f(1), f(2), \ldots, f(N) \text {. }
$$

Oznaczmy przez $N(\gamma)$ liczbę wyrazów spełniających warunek

$$
f(k)-[f(k)]<\gamma, \quad \text { gdzie } \quad 0 \leqslant \gamma \leqslant 1 .
$$

Mówimy, że ciag $f$ ma ekwipartycje, jeśli

$$
\lim _{N \rightarrow \infty} \frac{N(\gamma)}{N}=\gamma
$$

DefinicJa 2. Niech będzie $m$ ciagów:

$$
\begin{gathered}
f_{1}(1), f_{1}(2), \ldots, f_{1}(N), \\
f_{2}(1), f_{2}(2), \ldots, f_{2}(N), \\
\ldots \ldots \ldots \ldots \\
f_{m}(1), f_{m}(2), \ldots, f_{m}(N) .
\end{gathered}
$$

Oznaczmy przez $N\left(\gamma_{1}, \gamma_{2}, \ldots, \gamma_{m}\right)$ liczbe kolumn tego zespołu ciagów, spełniających dla każdego $k$ od 1 do $m$ warunek

$$
f_{k}(j)-\left[f_{k}(j)\right]<\gamma_{k},
$$

gdzie $0 \leqslant \gamma_{k} \leqslant 1$. Mówimy, że zespól ciagów $f_{1}, f_{2}, \ldots, f_{m}$ ma ekwipartyoje, jesli

$$
\lim _{N \rightarrow \infty} \frac{N\left(\gamma_{1}, \gamma_{2}, \ldots, \gamma_{m}\right)}{N}=\gamma_{1} \gamma_{2} \ldots \gamma_{m} .
$$

Jeśli zespół ciągów $f_{1}, f_{2}, \ldots, f_{m}$ ma ekwipartycje, to każdy jego podzespół ma również ekwipartycję, co otrzymamy od razu nakładaj̨̨e warunek $\gamma=1$ na wszystkie $f$ poza podzespołem.

TWLERDzENIE I. Jesili $\theta$ jest dowolna liczba niewymierna, to ciag

$$
\theta, 2 \theta, 3 \theta, \ldots, N \theta
$$

ma elkwipartycje.

Twierdzenie to zostało dowiedzione w roku 1909 przez Sierpińskiego, jak również przez Bohla, a w 1916 r. Weyl podał prosty dowód opierający się na metodzie majacej szersze zastosowanie $\left(^{1}\right)$.

(1) Patrz J. F. Koksma, Diophantische Approximationen, Berlin 1936, str. 92. 
TwIERDzNIE II (szczególny przypadek innego twierdzenia Weyla $\left({ }^{2}\right)$ ). Zespól ciagów

$$
\begin{aligned}
& \theta_{1}, 2 \theta_{1}, \ldots, N \theta_{1}, \\
& \theta_{2}, 2 \theta_{2}, \ldots, N \theta_{2}, \\
& . . . . . \cdot \\
& \theta_{m}, 2 \theta_{m}, \ldots, N \theta_{m}
\end{aligned}
$$

ma ekwipartycje, jeśli dla dowolnego zespotu liczb calkowitych $h_{1}, h_{2}, \ldots, h_{m}$ nie równych jednocześnie zeru wyrażenie

jest liczba niewymierna.

$$
h_{1} \theta_{1}+h_{2} \theta_{2}+\ldots+h_{m} \theta_{m}
$$

ROWWOWAŻNIK TWIERDZENIA II. Jezeli wszystkie liczby $\theta_{1}, \theta_{2}, \ldots, \theta_{m} s q$ niewymierne $i$ żadna $z$ nich nie jest funkcja liniowa o wspólczynnikach wymiernych innych $\theta$, to zespót (2) ( $i$ każdy jego podzespót) ma ekwipartycje.

$\mathrm{Z}$ twierdzenia I wyprowadzamy teraz następujące

TWIERDZENIE III. Oznaczmy przez S liczbe wyrazów ciagu (1), które spelniaja warunek (a) lub (b):

$$
\begin{array}{ll}
j \theta-[j \theta]<\gamma, & (j+1) \theta-[(j+1) \theta] \geqslant \gamma, \\
j \theta-[j \theta] \geqslant \gamma, & (j+1) \theta-[(j+1) \theta]<\gamma .
\end{array}
$$

Stosunek $\mathbb{S} / N$ zmierza do granioy określonej dalej wzorami (3), (4) $i$ (5), $g d y \rightarrow \infty$.

Oznaczmy $\theta-[\theta]$ przez $\theta^{\prime}$. Latwo zauważyé, że

$$
j \theta-[j \theta]=j \theta^{\prime}-\left[j \theta^{\prime}\right] .
$$

Wobec tego w warunkach (a) i (b) można podstawić $\theta^{\prime}$ zamiast $\theta$, przy czym $\theta^{\prime}<1$. Można też bez uszczuplenia ogólności dowodu założyé, że $\gamma \leqslant \frac{1}{2}$.

Twierdzenia latwo jest dowieść graficznie. Jeśli $j \theta^{\prime}$ odkładać będziemy na kole o obwodzie 1 od punktu zerowego w kierunku ruchu wskazówki zegara, to $j \theta^{\prime}-\left[j \theta^{\prime}\right]$ jest odstępem punktu $j \theta^{\prime}$ od zera. Warunki (a) i (b) oznaczają, że jeśli punkt $j \theta^{\prime}$ leży w obrębie luku $\gamma$, to punkt $(j+1) \theta^{\prime}$ leży poza nim lub vice versa. Bez uszczuplenia ogólności dowodu można założyé, że zero leży na początku tuku $\gamma$. Rozpatrzymy trzy przypadki:
(A)

$$
\begin{aligned}
\theta^{\prime} & <\gamma, \\
\gamma \leqslant \theta^{\prime} & <1-\gamma, \\
\theta^{\prime} & \geqslant 1-\gamma,
\end{aligned}
$$

którym odpowiadają rysunki 1,2 i 3.

(") Ibidem, str. 93 . 
Na rysunkach punkty $j \theta^{\prime}$ na łukach zaznaczonych grubą linią odpowiadają, jak tego dowiedziemy dalej, warunkom (a) lub (b).

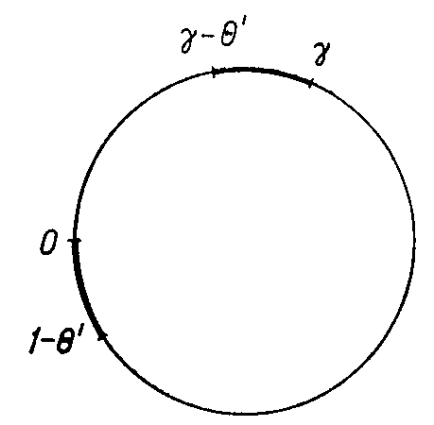

$2 M-235$

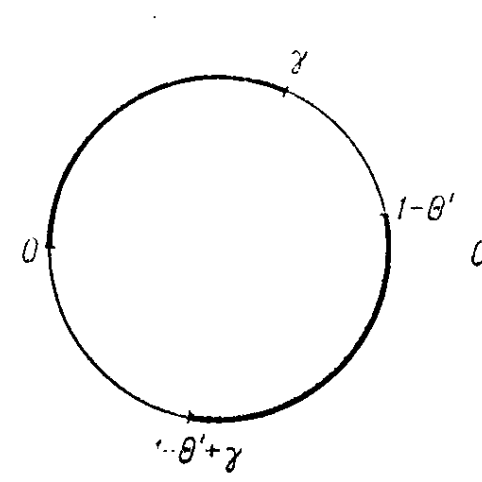

$2 M-236$

Rrs. 2

Ris. 1

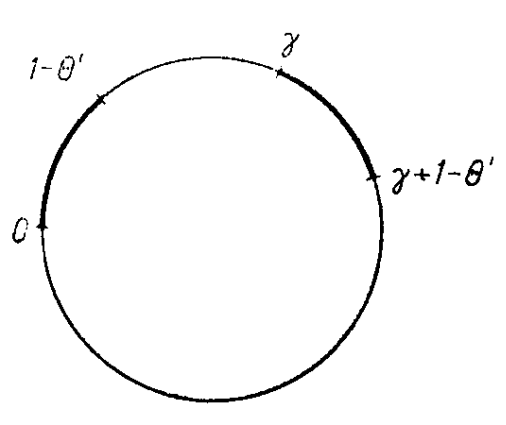

$Z M-237$

Ris. $3 \div$ :

Rozważmy przy padek (A). Jeżeli punkt $j \theta^{\prime}$ leży w obrębie łuku $\left(1, \gamma\right.$, to punkt $(j+1) \theta^{\prime}$ leży poza nim wtedy i tylko wtedy, gdy punkt $j \theta^{\prime}$ leży na luku $\gamma-\theta^{\prime}, \gamma$. Tak samo, jeżeli punkt $j \theta^{\prime}$ leży $w$ obrębie łaku $\gamma, 1-\theta^{\prime}$, to $(j+1) \theta^{\prime}$ lézy poza nim wtedy i tylko wtedy, gdy $j \theta^{\prime}$ leży na luku 1- $\theta^{\prime}, \mathbf{0}$.

Z twierdzenia I wynika, że w tym przypadku

$$
\lim _{N \rightarrow \infty} \frac{S}{N}=2 \theta^{\prime}
$$

W przypadku (B), jeśli punkt $j \theta^{\prime}$ leży w obrębie łuku $0, \gamma$, to $(j+1) \theta^{\prime}$ leży poza nim. Jeśli $j \theta^{\prime}$ leży w obrębie tuku $\gamma, 0$, to $(j+1) \theta^{\prime}$ leży wtedy i tylko wtedy poza nim, gdy punkt $j \theta^{\prime}$ leży na tuku $1-\theta^{\prime}, 1-\theta^{\prime}+\gamma$. W tym przypadku mamy

$$
\lim _{N \rightarrow \infty} \frac{\boldsymbol{B}}{N}=2 \gamma
$$

Wreszcie w przypadku (C), jeśli punkt j $\theta^{\prime}$ leży w obrębie luku $0, \gamma$, to $(j+1) \theta^{\prime}$ leży wtedy i tylko wtedy poza nim, gdy punkt $j \theta^{\prime}$ leży na luku $0,1-\theta^{\prime}$. Tak samo, jeśli $j \theta^{\prime}$ leży w obrębie łuku $\gamma, 0$, to $(j+1) \theta^{\prime}$ leży wtedy i tylko wtedy poza nim, gdy $j \theta^{\prime}$ leży na luku $\gamma, \gamma+1-\theta^{\prime}$. W tym przypadku otrzymujemy

$$
\lim _{N \rightarrow \infty} \frac{S}{N}=2\left(1-\theta^{\prime}\right)
$$

W ten sposób dowiedliśmy, że gdy $N \rightarrow \infty$, to stosunek ilości $\mathbb{S}$ wyrazów ciagu $\theta, 2 \theta, \ldots, N \theta$, spehniających warunek (a) lub (b), do 
ogólnej liczby wyrazów $N$ zmierza do granicy określonej wzorami (3), (4) i (5), która oznaczymy przez $\delta$ :

$$
\lim _{N \rightarrow \infty} \frac{S}{N}=\delta
$$

Z twierdzeń III oraz II wyprowadzamy następujące

TwIERDzenIE IV. Oznaczmy przez $\mathbb{S}_{1,2, \ldots, N}$ liczbe kolumn zespolu. ciagów (2) spelniajacych dla każdego $k$ od 1 do $m$ warunek (a) lub (b):

$$
\begin{array}{ll}
j \theta_{k}-\left[j \theta_{k}\right]<\gamma, & (j+1) \theta_{k}-\left[(j+1) \theta_{k}\right] \geqslant \gamma, \\
j \theta_{k}-\left[j \theta_{k}\right] \geqslant \gamma, & (j+1) \theta_{k}-\left[(j+1) \theta_{k}\right]<\gamma .
\end{array}
$$

Oznaczmy dalej liczbe wyrazów spelniajqcych warunek (a) lub (b) w ciagu $k$ prżez $S_{k}$ oraz $\lim _{N \rightarrow \infty}\left(\mathbb{S}_{k} / N\right)$ przez $\delta_{k}$. Mamy wtedy

$$
\lim _{N \rightarrow \infty} \frac{S_{1,2, \ldots, N}}{N}=\delta_{1} \delta_{2} \ldots \delta_{m}
$$

(Będzie to stuszne również dla każdego podzespolu zespołu ciagów (2).)

Wyobraźmy sobie $m$ kół o obwodach 1 . Na $k$-tym kole punkty $j \theta_{k}$ spełniające warunek (a) lub (b) leża na dwóch określonych łukach o łącznej dhugości $\delta_{k}$. Z twierdzenia II wynika, że stosunek liczby tych $j$, dla których warunek (a) lub (b) spełniony jest na wszystkich okręgach, tzn. $\$_{1,2, \ldots, N}$, do $N$ równa się iloczynowi $\delta_{1} \delta_{2} \ldots \delta_{m}$.

TWIERDZENIE V. Jeśli $\left|1-2 \gamma_{k}\right| \leqslant \alpha<1$ oraz $\theta_{k}^{\prime} \geqslant \beta>0,1-\theta_{k}^{\prime} \geqslant$ $\geqslant \beta>0,\left|\frac{1}{2}-\theta_{k}^{\prime}\right| \geqslant \beta>0$, gdzie a $i \beta$ sq to liczby state, to $\left|1-2 \delta_{k}\right| \leqslant \lambda<1$, gdzie $\lambda$ jest znowu liczbq stata.

Dowód. Należy przede wszystkim zaznaczyć, że bez uszczuplenia ogólności twierdzenia możemy założyć $\alpha>\frac{1}{2}$ i $\beta \ll \frac{1}{4}$.

Dla trzech przypadków rozpatrywanych w dowodzie twierdzenia III mamy dla $\delta_{k}$ i $\left|1-2 \delta_{k}\right|$ (zakładając jak w tamtym dowodzie, że $\gamma_{k} \leqslant \frac{1}{2}$ )

\begin{tabular}{cc|c|c} 
& Przypadek & $\delta_{k}$ & \multicolumn{1}{|c}{$\left|1-2 \delta_{k}\right|$} \\
\hline (A) $\quad \theta_{k}^{\prime}<\gamma_{k} \leqslant \frac{1}{2}$ & $2 \theta_{k}^{\prime}$ & $\left|1-4 \theta_{k}^{\prime}\right|$ \\
(B) $\quad \gamma_{k} \leqslant \theta_{k}^{\prime}<1-\gamma$ & $2 \gamma_{k}$ & $\left|1-4 \gamma_{k}\right|$ \\
(C) $\quad \frac{1}{2} \leqslant 1-\gamma_{k} \leqslant \theta_{k}^{\prime}$ & $2\left(1-\theta_{k}^{\prime}\right)$ & $\left|1-4\left(1-\theta_{k}^{\prime}\right)\right|$
\end{tabular}

W przypadkach (A) i (C) z warunków

$$
\theta_{k}^{\prime} \geqslant \beta<\frac{1}{4}, \quad 1-\theta_{k}^{\prime} \geqslant \beta<\frac{1}{4}, \quad\left|\frac{1}{2}-\theta_{k}^{\prime}\right| \geqslant \beta<\frac{1}{4}
$$


wynika, jak łatwo zauważyé, że

$$
\left|1-2 \delta_{k}\right| \leqslant 1-4 \beta<1 .
$$

W przypadku (B) z warunku $\left|1-2 \gamma_{k}\right| \leqslant \alpha$ i z nierówności $\gamma_{k} \leqslant \frac{1}{2}$ wynika, że

$$
\gamma_{k} \geqslant \frac{1}{2}(1-\alpha) .
$$

Następnie z warunku $\left|\frac{1}{2}-\theta_{k}^{\prime}\right| \geqslant \beta$ wynika, że

$$
\theta_{k}^{\prime} \leqslant \frac{1}{2}-\beta \quad \text { albo } \quad 1-\theta_{k}^{\prime} \leqslant \frac{1}{2}-\beta \text {. }
$$

Poza tym mamy $\gamma_{k} \leqslant \theta_{k}$ az $1-\gamma_{k}>\theta_{k}^{\prime}$, czyli $\gamma_{k}<1-\theta_{k}^{\prime}$. Stąd wynika, $\dot{z} \mathrm{e}$

Mamy więc

$$
\gamma_{k} \leqslant \frac{1}{2}-\beta \text {. }
$$

$$
\frac{1}{2}(1-\alpha) \leqslant \gamma_{k} \leqslant \frac{1}{2}-\beta .
$$

Otrzymujemy stąd dla przypadku $\gamma_{k} \leqslant \frac{1}{4}$

$$
\left|1-4 \gamma_{k}\right| \leqslant 1-4(1-\alpha) / 2=2 \alpha-1<1,
$$

a dla przypadku $\gamma_{k}>\frac{1}{4}$

$$
\left|1-4 \gamma_{k}\right| \leqslant 1-4 \beta<1 .
$$

Stąd wynika, że jeśli $\lambda$ jest równe większej z liczb $2 \alpha-1$ i $1-4 \beta$, to mamy

$$
\left|1-2 \delta_{k}\right| \leqslant \lambda<1 .
$$

TwIerdzenIE VI. Twierdzenia III, IV $i \mathrm{~V}$ pozostaja $w$ mocy, jeśli warunki (a) $i$ (b) przybieraja ogólniejsza postaé

$$
\begin{array}{ll}
j \theta_{k}-\left[j \theta_{k}\right]<\gamma_{k}, & (j+a) \theta_{k}-\left[(j+a) \theta_{k}\right] \geqslant \gamma_{k}, \\
j \theta_{k}-\left[j \theta_{k}\right] \geqslant \gamma_{k}, & (j+a) \theta_{k}-\left[(j+a) \theta_{k}\right]<\gamma_{k},
\end{array}
$$

gdzie a jest liczba dodatnia calkowita, $i$ jeśli $w$ twierdzeniu $\mathrm{V}$ zastapimy $\theta^{\prime}$ przez $\theta_{k}^{(a)}=a \theta_{k}-\left[a \theta_{k}\right]$.

Dowody tych twierdzeń przeprowadzić można jak wyżej, zastępujq̨e $\theta^{\prime}$ przez $\theta_{k}^{(a)}=a \theta_{k}-\left[a \theta_{k}\right]$. 
М. КА ЛЕДКИН (Варmава)

\section{МЕХАНИСТИЧЕСКАЯ МОДЕЛЬ СЛУЧАЙНГО ЯВЛЕНИЯ \\ P E 3 ○ $\mathrm{M}$ E}

Пусть будет дано $m$ часов, циферблаты которых имеют периметр равныи единице и разделены на два сегмента - черный и белый. Каждые иа часов имеют только одну стрелку, конед которой движется соответственио со скоростью $\theta_{1}, \theta_{2}, \ldots, \theta_{m}$. Через каждую единицу времени проверяется, находятсп ли стрелни всех $m$ часов в черном или же в белом сегменте. Если число черных сегментов четно, то записывается $\boldsymbol{A}$, если же нечетно, записывается $\boldsymbol{B}$.

Пусть $(\boldsymbol{A})_{\boldsymbol{N}}$ овначает частоту появлепия события $\boldsymbol{A}$ в $\boldsymbol{N}$ наблюдениях, a $\left(A_{i}, A_{j}+a\right) N m$ - частоту появления комбинации $A A$ в наблюдениях с номерами $j$ и $j+a$. Аналогично определены обозначения $(B)_{N m},\left(B_{j}, B_{j+a}\right)_{N m},\left(A_{f}, B_{j+a}\right)_{N m}$, $\left(B_{j}, A_{j+a}\right)_{N m}$.

При некоторых предположениях относияельно скоростей $\theta_{1}, \theta_{2}, \ldots, \theta_{m}$ и ири предположении, что длина черного сегмента на $k$-ьх часах $p_{k}$ удовлет. воряет условию $\left|1-2 p_{k}\right| \leqslant \alpha<1$, где $\alpha$ константа, автор получает следуюшие теоремы :

$$
\lim _{m \rightarrow \infty} \lim _{N \rightarrow \infty}(A)_{N m}=\lim _{m \rightarrow \infty} \lim _{N \rightarrow \infty}(B)_{N m}=\frac{1}{2}
$$

(2) $\lim _{m \rightarrow \infty} \lim _{N \rightarrow \infty}\left(A_{j} A_{j+a}\right) . N m=\lim _{m \rightarrow \infty} \lim _{N \rightarrow \infty}\left(B_{j} B_{j+a}\right)_{N m}=$

$$
=\lim _{m \rightarrow \infty} \lim _{N \rightarrow \infty}\left(A_{j} B_{j+a}\right)_{N m}=\lim _{m \rightarrow \infty} \lim _{N \rightarrow \infty}\left(B_{j} A_{j+a}\right)_{N m}=1
$$

Это вначит, ипо $j$-ое и $(j+a)$-ое наблюдения асимлтотически независимы с произ. өольной степеньо точности, ковда т достаточно велико.

(3) ј-ое наблюдение асимнтотически независимо в этом смысле от всех а предыдучих наблюдений.

В конце статьи автор покавывает, что при помощи рассматриваемой детөр. министской системы можно описать появление rouge и поir в рулетке.

\section{KAL ECKI (Wargzawa)}

\section{MECHANTSTIC MODEL OF A RANDOM PHENOMENON}

\section{S U M M A R Y}

Let $m$ denote the number of watches whose faces have a circumference equal to unity and are divided into two segments, a black and a white one. Each of the watches has only one hand, whose point moves with the velooity $\theta_{1}, \theta_{2}, \ldots, \theta_{m}$. We check at intervals of a unit of time all the $m$ watohes to seo whether their hands are in the black or in the white field. If the number of black fields is even, we record $A$; if it is odd, we record $B$.

Let $(A)_{N m}$ denote the frequency of the occurrenoe of $A$ in $N$ observations, and $\left(A_{f} A_{j+a}\right)_{\mathrm{Nm}}$ the frequeney of the ocenrrence of the combination $A A$ in the $j$-th 
and the $(j+a)$-th observations. In a similar way we define the symbols $(B)_{N m}$ and $\left(B_{f} B_{f+a}\right)_{N m},\left(A_{f} B_{f+a}\right)_{N m}$ and $\left(B_{f} A_{f+a}\right)_{N m}$.

Under certain assumptions regarding the velocities $\theta_{1}, \theta_{2}, \ldots, \theta_{m}$ and under the assumption that the length $p_{k}$ of the are of the black segment on the $k$-th watch satisfies the condition $\left|1-2 p_{k}\right| \leqslant \alpha<1$ where $a$ is fixed, the author obtains the following theorems:

$$
\lim _{m \rightarrow \infty} \lim _{N \rightarrow \infty}(A)_{N m}=\lim _{m \rightarrow \infty} \lim _{N \rightarrow \infty}(B)_{N m}=\frac{1}{2} ;
$$

(2) $\lim _{m \rightarrow \infty} \lim _{N \rightarrow \infty}\left(A_{j} A_{j+a}\right)_{N m}=\lim _{m \rightarrow \infty} \lim _{N \rightarrow \infty}\left(B_{j} B_{j+a}\right)_{N m}=$

$$
=\lim _{m \rightarrow \infty} \lim _{N \rightarrow \infty}\left(A_{j} B_{j+a}\right)_{N m}=\lim _{m \rightarrow \infty} \lim _{N \rightarrow \infty}\left(B_{j} A_{j+a}\right)_{N m}=\frac{1}{4},
$$

which means that the $j$-th and the $(j+a)$-th observations are asymptotically independent with any degree of approximation if $m$ is sufficiently large.

(3) The $j$-th observation is, in the same manner, asymptotically independent of all the a observations preceding it.

Finally the author shows that by means of the deterministic system considered it is possible to desoribe the occurrence of rouge and noir in the roulette. 